\title{
Planning, prospective memory, and decision-making: three challenges for hierarchical predictive processing models
}

\author{
Demis Basso ${ }^{1,2 *}$ \\ ${ }^{1}$ Faculty of Education, Free University of Bozen-Bolzano, Bolzano, Italy \\ ${ }^{2}$ Centro di Neuroscienze Cognitive Applicate, Rome, Italy \\ *Correspondence: demis.basso@unibz.it
}

Edited by:

Axel Cleeremans, Université Libre de Bruxelles, Belgium

Reviewed by:

Axel Cleeremans, Université Libre de Bruxelles, Belgium

Shimon Edelman, Cornell University, USA

\section{A commentary on}

Whatever next? Predictive brains, situated agents, and the future of cognitive science by Clark, A. (2012). Behav. Brain Sci. (in press).

The present commentary on Clark (in press) will emphasize and discuss the role that high processing related to action planning may have in Bayesian predictive processing and will suggest possible directions for managing the issue.

Clark agrees to define planning as follows: "we imagine a future goal state as actual, then use Bayesian inference to find the set of intermediate states (which can now themselves be whole actions) that get us there" (\$1.5). Although this definition was reported to corroborate the unified vision of sensor processing, motor control, and planning suggested by Toussaint (2009), it does not correspond to the current representation of the planning process in two aspects.

The first aspect entails the hierarchical vision of a plan as a succession of intermediate states. Research on planning (Basso et al., 2001; Phillips et al., 2001) has shown that the future goal state created in the beginning is accurate only in some particular circumstances (i.e., when both the task and algorithm are well-defined). In most cases, people are used to facing underspecified tasks in which a future goal state cannot be employed to derive the intermediate states (see Goel and Grafman, 2000). The first plan created is a general sketch of the intentions, a blurred image of the desired goal created by a feedforward prediction, which is successively refined through a continuous interaction between action and perception (Basso and Olivetti Belardinelli, 2006; Cisek, 2007). When the hierarchical predictive processing (HPP) presented by Clark is applied to the planning of motor sequences, it is undoubtedly convincing, as it explains the sensorimotor loop in an efficient way. However, the same mechanism may be too limited when applied to the planning of complex actions sequences. For example, in a planning task such as the Traveling Salesperson Problem (TSP: MacGregor and Ormerod, 1996), participants changed the initially planned strategy during task execution (Basso et al., 2006). Moreover, Cazzato et al. (2010) have demonstrated that a proficient performance is related to cognitive flexibility (shown while reconsidering the strategy chosen in the beginning) and not to the amount of information retrieved by ocular movements. That is, the overall organized behavior must be considered as more important than the summation of single actions.

The second aspect entails the timing in which the plan and (sequence of) action(s) may occur. In a stimulusresponse paradigm a plan is created and promptly used because the action must be implemented as soon as possible. However, in many real-world situations, events develop in time and the planned actions must be postponed in order to be executed at the appropriate moment. Gärling (1994) has provided an example of everyday planning using a fictitious environment in which participants must organize a trip through several errands like stores with different opening hours. This delay between the plan and its implementation is also shared with other cognitive processes such as prospective memory (PM: Kliegel et al., 2008).

PM requires that a planned action should be executed only whenever the circumstances fulfill the conditions, which were commonly not present when the plan was created. In a standard scenario, a plan should be kept in mind while a person is involved in other ongoing activities, until the activating conditions are satisfied. At that moment, the person should inhibit the ongoing activity, switch to the prospective activity, and execute the plan. During the ongoing activity, some lure stimuli (distracters, see for PM: Bisiacchi et al., 2011) could share certain commonalities with the activating cues, but not enough to satisfy all the conditions. In these cases, according to HPP, the prospective action would increase its activation value and the probability of being executed as well, whereas it should be inhibited instead. HPP models are also required to account for the inhibition generated by long-lasting intentions. In the present state of the art, HPP-based models are outstanding in producing a response to a stimulus, but this response is locked in time to the stimulus itself (i.e., it is simultaneous to or must follow the stimulus as closely as possible). Providing explanations also for the realization of plans with delayed actions would be a good benchmark for determining the effective legitimacy of Clark's approach.

One attempt of this kind has been proposed by Shadlen et al. (2008) in decisionmaking. According to their accumulator model, decisions are taken when the accumulated evidence promoting a specific choice exceeds a certain threshold value (determined by prior information and 
costs). Abstract decisions (i.e., those producing a plan) are essentially aimed at creating rules instead of actions. It is important to highlight that, with respect to Clark's model, Shadlen's includes some components of the Bayesian inference, but it avoids using its major assumption, the posterior probability. Higher processing falls beyond the aim of Clark's target article, but it is central in its relevance for a model aiming at describing human processing. It is not that motor planning is more or less important than other higherlevel planning, but they both show the same level of importance and need to be explained.

A unified mechanism for managing input-output processes is undoubtedly efficient and successful in species evolution. Therefore, it is likely that it is shared with many animals, too. However, since human beings have developed more complex behaviors and processes with respect to those managed by other animals, HPP must be shown to be flexible enough to (1) manage high levels of information (if it is the only mechanism), or (2) collaborate with other mechanisms of information processing. Clark is aware of this possible limitation, as he posed it in terms of open questions. The mechanism of predictive processing could be sufficiently powerful to be successfully applied to higher processing, as suggested by Cisek and Kalaska (2010, p. 276) too, but largescale non-hierarchical mechanisms (able to recursively manage several processing systems) must also be hypothesized. Evidence from neuroscience enlightened that magnetic stimulation produces different effects, which are dependent on initial conditions of the cell assembly (Hoshi et al., 2000; Silvanto et al., 2008). State-dependent cell assemblies provide the cognitive system with flexibility that is likely to account for high-level (longterm) processes such as planning, PM, and decision-making.

\section{REFERENCES}

Basso, D., Bisiacchi, P. S., Cotelli, M., and Farinello, C. (2001). Planning times during Travelling Salesman's problem: differences between closed head injury and normal subjects. Brain Cogn. 46, 38-42.

Basso, D., Lotze, M., Vitale, L., Ferreri, F., Bisiacchi, P. S., Olivetti Belardinelli, M., et al. (2006). The role of prefrontal cortex in visuo-spatial planning: a repetitive-TMS study. Exp. Brain Res. 171, 411-415.

Basso, D., and Olivetti Belardinelli, M. (2006). The role of the feedforward paradigm in cognitive psychology. Cogn. Process. 7, 73-88.

Bisiacchi, P. S., Cona, G., Schiff, S., and Basso, D. (2011). Modulation of fronto-parietal network in event-based prospective memory: an rTMS study. Neuropsychologia 49, 2225-2232.

Cazzato, V., Basso, D., Cutini, S., and Bisiacchi, P. S. (2010). Gender differences in visuospatial planning: an eye movements study. Behav. Brain Res. 206, 177-183.

Cisek, P. (2007). Cortical mechanisms of action selection: the affordance competition hypothesis. Philos. Trans. R. Soc. Lond. B Biol. Sci. 362, 1585-1599.

Cisek, P., and Kalaska, J. F. (2010). Neural mechanisms for interacting with a world full of action choices. Annu. Rev. Neurosci. 33, 269-298.

Clark, A. (2012). Whatever next? Predictive brains, situated agents, and the future of cognitive science. Behav. Brain Sci. (in press).

Gärling, T. (1994). Processing of time constraints on sequence decisions in a planning task. Eur. J. Cogn. Psychol. 6, 399-416.

Goel, V., and Grafman, J. (2000). Role of the right prefrontal cortex in ill-structured planning. Cogn. Neuropsychol. 17, 415-436.
Hoshi, E., Shima, K., and Tanji, J. (2000). Neuronal activity in the primate prefrontal cortex in the process of motor selection based on two behavioral rules. J. Neurophysiol. 83, 2355-2373.

Kliegel, M., McDaniel, M. A., and Einstein, G. O. (2008). Prospective Memory: Cognitive, Neuroscience, Developmental, and Applied Perspectives. New York, NY: Lawrence Erlbaum Associates.

MacGregor, J. N., and Ormerod, T. C. (1996). Human performance on the travelling salesman problem. Percept. Psychophys. 58, 527-539.

Phillips, L. H., Wynn, V. E., McPherson, S., and Gilhooly, K. J. (2001). Mental planning and the Tower of London task. Q. J. Exp. Psychol. A 54, 579-598.

Shadlen, M. N., Kiani, R., Hanks, T. D., and Churchland, A. K. (2008). "Neurobiology of decision making: an intentional framework," in Better than Conscious? Decision Making, the Human Mind, and Implications for Institutions, eds C. Engel and W. Singer (Cambridge, MA: MIT Press), 71-101.

Silvanto, J., Muggleton, N. G., and Walsh, V. (2008). State dependency in brain stimulation studies of perception and cognition. Trends Cogn. Sci. 12, 447-454.

Toussaint, M. (2009). Probabilistic inference as a model of planned behavior. Künstliche Intelligenz 3, 23-29.

Received: 31 August 2012; accepted: 31 December 2012; published online: 18 January 2013.

Citation: Basso D (2013) Planning, prospective memory, and decision-making: three challenges for hierarchical predictive processing models. Front. Psychology 3:623. doi: $10.3389 /$ fpsyg.2012.00623

This article was submitted to Frontiers in Theoretical and Philosophical Psychology, a specialty of Frontiers in Psychology.

Copyright (c) 2013 Basso. This is an open-access article distributed under the terms of the Creative Commons Attribution License, which permits use, distribution and reproduction in other forums, provided the original authors and source are credited and subject to any copyright notices concerning any third-party graphics etc. 